\title{
Fournier's gangrene: Ultrasound or Computed Tomography?
}

\author{
Nishant Gupta1, Kenneth M. Zinn', Itisha Bansal², Robert Weinstein ${ }^{3}$ \\ ${ }^{1}$ Ultrasound Division, Department of Radiology and Imaging, ${ }^{2}$ Department of Internal Medicine, ${ }^{3}$ Department of \\ Urology, St. Vincent's Medical Center, Bridgeport, Connecticut, USA
}

\section{To the Editor,}

We read the case report published in the Medical Ultrasonography [1], on "Fournier's Gangrene" by Matilsky et al with academic interest. It is appreciable that the authors have presented this rare case of necrotizing fasciitis using bedside ultrasound. However, CT scan of the abdomen and pelvis should be considered next for planned surgical management. We agree to the fact that the diagnosis of Fournier's gangrene can be made with bedside ultrasound with a very high sensitivity [2,3]; however CT can be more specific not only in confirming the diagnosis, but also in evaluating the disease extent $[4,5]$. CT scan of the abdomen and pelvis can also unfold the underlying cause, as it is rare for Fournier's gangrene to be idiopathic [4-6]. We came across a 52 year-old male diabetic patient, who presented with a 4 day history of left scrotal pain and heaviness in the ED, and was diagnosed as Fournier's gangrene on bedside sonography (fig 1). The patient immediately underwent a CT scan of the abdomen and pelvis, which revealed subcutaneous air in the left scrotal sac, extending to the left inguinal canal, without any other focus of infection (fig 2). The CT scan solidified the plan of surgeons to perform isolated left scrotectomy, thus decreasing the overall surgical morbidity. At surgery, approximately $50 \mathrm{ml}$ of foul smelling fluid was drained. Currently, the patient is planned for

Received 08.09.2014 Accepted 09.09.2014

Med Ultrason

2014, Vol. 16, No 4, 389-390

Corresponding author: Nishant Gupta MD PDCC

Department of Radiology and Imaging,

St. Vincent's Medical Center,

2800 Main street, Bridgeport,

Connecticut 06460, USA.

Phone: 203-576-5533

Fax: 203-581-6513

Email: drngupta20@gmail.com

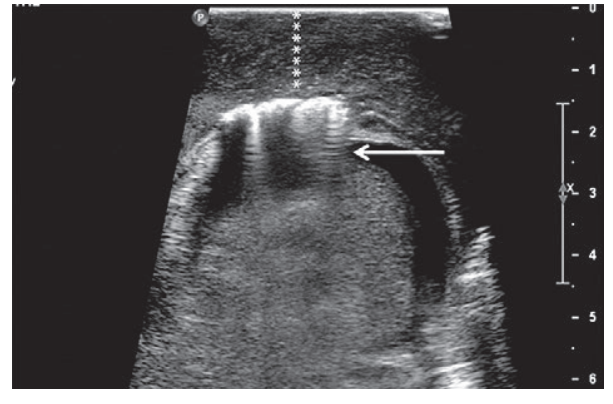

Fig 1. Transverse ultrasound of the left hemiscrotum reveals reverberation artifact from the scrotal wall (white arrow). Also note the thickened left scrotal wall (asterix).

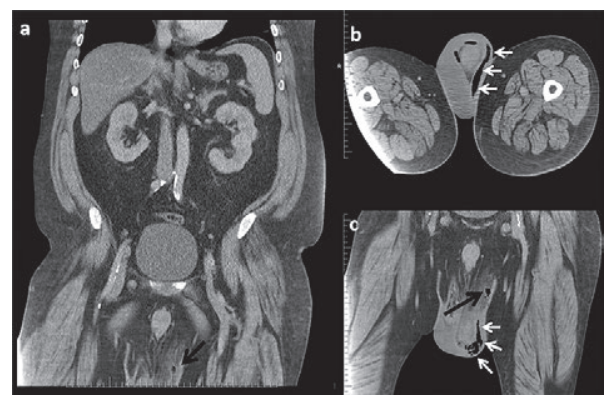

Fig 2. Coronal (a), axial (b) and sagittal (c) CT images of the abdomen and pelvis reveals subcutaneous air in the scrotal wall (white arrows). Note the extension of the air in the region of left inguinal canal (black arrow).

another debridement, before scrotal skin reconstruction can be performed.

The authors have very rightly described the fact, that the Fournier's gangrene can be diagnosed using bedside ultrasound, with a very high sensitivity, showing rever- 
beration artifact due to subcutaneous air in the non-dependent part of the scrotal wall $[2,3,7]$. However, CT can unleash the underlying pathology causing Fournier's gangrene, as well as can delineate the pathways of its spread, and thus can be of help in surgical planning, preventing another trip to the operating room for any unforeseen underlying pathology causing Fournier's gangrene. Timing is very important in the management of Fournier's gangrene and a planned protocol can benefit the patient.

We do recommend a bedside sonography to rule out Fournier's gangrene, due to its high sensitivity, and if sonography points to Fournier's gangrene, the patient should immediately undergo a CT scan of the abdomen and pelvis, to look for any primary focus of infection. CT should also be considered in a patient with extreme tenderness, due to the direct pressure on the scrotum, limiting complete evaluation. Scrotal ultrasound is a very sensitive modality in diagnosing and evaluating acute scrotum, however further evaluation with CT scan should especially be considered in case of Fournier's gangrene, to evaluate the underlying etiology and surgical planning.

This letter is written with the aim that readers of Medical Ultrasonography can appreciate the proposed plan for the patients with scrotal pain and swelling, with the concern for Fournier's gangrene.

\section{References}

1. Matilsky D, Lewiss RE, Whalen M, Saul T. Fournier's gangrene. Case report. Med Ultrason. 2014; 16: 262-263.

2. Yen ZS, Wang HP, Ma HM, Chen SC, Chen WJ. Ultrasonographic screening of clinically suspected necrotizing fasciitis. Acad Emerg Med 2002; 9: 1448-1451.

3. Butcher CH, Dooley RW, Levitov AB. Detection of subcutaneous and intramuscular air with sonography: a sensitive and specific modality. J Ultrasound Med 2011; 30: 791-795.

4. Rajan DK, Scharer KA. Radiology of Fournier's gangrene. AJR Am J Roentgenol 1998; 170: 163-168.

5. Amendola MA, Casillas J, Joseph R, Antun R, Galindez O. Fournier's gangrene: CT findings. Abdom Imaging 1994; 19: 471-474

6. Ash L, Hale J. CT findings in perforated rectal carcinoma presenting as Fournier's gangrene in the emergency department. Emerg Radiol 2005; 11: 295-297.

7. Feldman MK, Katyal S, Blackwood MS. US artifacts. Radiographics 2009; 29: 1179-1189. 